\title{
Efeitos de diferentes focos de atenção na performance motora de uma tarefa de agilidade em crianças
}

\author{
Effects of different foci of attention in the motor performance of a task \\ agility in children
}

\author{
Francisco de Assis Furtado de Oliveira1 ${ }^{1}$, Matheus Maia Pacheco², Ricardo Drews ${ }^{3 *}$ \\ ARTIGO ORIGINAL | ORIGINALARTICLE
}

\begin{abstract}
O objetivo do estudo foi investigar se instruções de foco externo de atenção comparado ao foco interno e sem foco determinado afetariam de forma diferente a performance motora de crianças. Adicionalmente, buscou-se mensurar a adoção das instruções prescritas sobre o foco de atenção pelos participantes. Vinte e três crianças, de ambos os sexos, com idade média de $10.04 \pm .06$ anos realizaram uma tarefa motora de agilidade em três condições diferentes: foco externo, foco interno e sem foco determinado. Estes realizaram cinco tentativas de prática de cada condição experimental em dias distintos e tiveram seus tempos de movimento registrados. Após cada tentativa, todos os participantes responderam a uma pergunta em relação ao foco de atenção utilizado. As análises apontaram superioridade da condição sem foco determinado e de foco interno em relação à condição foco externo. Em relação à adoção do foco de atenção, as crianças se utilizaram prioritariamente dos focos de atenção instruídos pelo experimentador. Porém, na condição sem foco determinado utilizaram o foco externo (65\%) na maioria das tentativas. Conclui-se que as instruções relacionadas ao foco de atenção externo não beneficiaram a performance motora de uma tarefa de agilidade em crianças.

Palavras-chaves: controle motor, desempenho motor, foco externo, foco interno, instruções, tempo de movimento
\end{abstract}

ABSTRACT

The aim of this study was to investigate whether instructions external focus of attention compared to internal focus and no particular focus differently affect motor performance of children. Additionally, we attempted to measure the adoption of the prescribed instructions on the focus of attention by the participants. Twenty-three children, of both sexes, mean age $10.04 \pm .06$ years performed a motor task agility in three different conditions: external focus, internal focus and no particular focus. They performed five trials of practice each experimental condition on different days and had their times recorded movement. After each trial, all participants answered a question regarding the focus of attention used. The analysis showed superiority of condition without specific focus and internal focus in relation to external focus condition. Regarding adoption of the focus of attention, the children used the priority focus of attention instructed by the experimenter. However, participants in the no particular focus condition used external focus $(65 \%)$ in most of the attempts. We conclude that the instructions related to the external focus of attention not benefited the motor performance of a task agility in children. Keywords: motor control, motor performance, external focus, internal focus, instructions, movement time

Artigo recebido a 18.12.2013; Aceite 05.05.2014

${ }^{1}$ Escola Superior de Educação Física, Universidade Federal de Pelotas, Brasil

${ }^{2}$ Pennsylvania State University, Estados Unidos da América

${ }^{3}$ Escola de Educação Física e Esporte, Universidade de São Paulo, Brasil

* Autor correspondente: Escola de Educação Física e Esporte - Universidade de São Paulo, Av. Prof. Mello Moraes, 65, Cidade Universitária, CEP 05508-030 São Paulo, SP - Brasil; E-mail: ricardodrews@hotmail.com 


\section{INTRODUÇÃO}

Um crescente número de estudos tem demonstrado que a eficácia na performance e aprendizagem motora tem grande dependência do foco de atenção induzido (para uma revisão, ver Wulf, 2013). Estudos sobre performance motora apontam que instruções que dirigiram a atenção dos indivíduos para os efeitos de seus movimentos no ambiente - foco externo - têmse mostrado superiores quando comparadas as instruções dirigidas a aspetos do padrão de movimento - foco interno (Freudenheim, Wulf, Madureira, Pasetto, \& Corrêa, 2010; Kal, van der Kamp, \& Houdijk, 2013; Marchant, Greig, Bullough, \& Hitchen, 2011; Schlesinger, Porter, \& Russell, 2012).

Uma gama de evidências dos benefícios do foco de atenção externo na performance motora encontram-se em análises com a população adulta (Lohse, Sherwood, \& Healy, 2010; Makaruk, Porter, Czaplicki, Sadowski, \& Sacewicz, 2012; Schücker, Hagemann, Strauss, \& Völker, 2009; Wulf, Dufek, Lozano, \& Pettigrew, 2010). Poucos estudos, entretanto, foram realizados com crianças (Olivier, Palluel, \& Nougier, 2008), havendo um número escasso de indícios demonstrando os efeitos do foco de atenção externo da performance motora nessa faixa etária. No entanto, se levarmos em conta os resultados encontrados na fase de prática dos estudos que analisaram os efeitos do foco de atenção na aprendizagem motora em crianças, encontramos resultados contraditórios sobre os benefícios do foco de atenção externo nessa faixa etária (Abdollahipour, Bahram, Shafizadeh, \& Khalaji, 2011; Chiviacowsky, Wulf, \& Avila, 2013; Emanuel, Jarus, \& Bart, 2008; Wulf, Chiviacowsky, Schiller, \& Avila, 2010). Especificamente, Abdollahipour, Bahram, Shafizadeh, e Khalaji (2011) verificaram menor número de erros na realização de uma tarefa de drible no futebol quando o grupo realizou a tarefa com instrução de foco interno. Por outro lado, Wulf, Chiviacowsky, Schiller, e Avila (2010) não verificaram diferenças entre grupos que receberam diferentes induções de foco de atenção externo e interno em uma tarefa de arremesso de bola.
Resultados semelhantes foram encontrados por Chiviacowsky, Wulf, e Avila (2013) e Emanuel, Jarus, e Bart (2008).

Uma hipótese para os resultados encontrados seria a de diferentes efeitos do foco de atenção nos primeiros níveis de aprendizagem (Fitts \& Posner, 1967; Gentile, 1972). Alguns estudos têm demonstrado efeitos distintos do foco de atenção em adultos iniciantes e habilidosos, sendo questionado se foco de atenção externo traz benefícios para a performance motora de participantes iniciantes (Beilock, Bertenthal, Mccoy, \& Carr, 2004; Beilock, Carr, MacMahon, \& Starkes, 2002; Ford, Hodges, \& Williams, 2005). Crianças, devido ao nível de desenvolvimento no qual se situam, passam por um período de menor proficiência nas habilidades em relação a adultos e carregam consigo menor experiência (Jerry R. Thomas, Lee, \& Thomas, 1988). Tendo por base que a distribuição da atenção, além de poder variar em função da complexidade da tarefa e das instruções fornecidas ao indivíduo, pode ser influenciada pelo seu nível de habilidade (Ivry, 1996; Shiffrin, 1988), se torna questionável se instruções induzindo a utilização do foco externo seriam eficientes na performance motora de crianças, como é encontrado na população de adultos.

Aliado a isto, a adoção do foco de atenção instruído necessita ser mensurada para uma melhor compreensão dos efeitos que a instrução sobre o foco de atenção na performance. Entretanto, poucos estudos controlaram a adoção do foco solicitado (Bell \& Hardy, 2009; Ford, Hodges, \& Williams, 2009; Porter, Nolan, Ostrowski, \& Wulf, 2010; Stoate \& Wulf, 2011). Assim, também se encontram incertezas relacionadas à adoção do foco instruído como se há tendências de utilização do foco em um grupo sem instrução direta. Apesar da especulação que indivíduos do grupo sem indução de foco tendem a escolher o foco interno (Wulf, 2007), Porter, Nolan, Ostrowski, e Wulf (2010) não encontraram foco definido para este grupo em adultos - e ainda encontraram adoção de diferentes instruções de foco utilizados em alguns casos. 
Portanto, o objetivo do presente estudo foi investigar se instruções de foco externo de atenção comparado ao foco interno e sem foco determinado afetariam de forma diferente a performance motora de crianças. Adicionalmente, foi verificado qual foco de atenção foi adotado pelos participantes em cada condição de prática, com intuito de mensurar a adoção das instruções, o foco de atenção utilizado na condição sem foco determinado e a frequência de troca de foco em cada condição experimental.

\section{MÉTODO}

\section{Amostra}

Participaram voluntariamente do estudo 23 crianças de ambos os sexos (14 meninos e 9 meninas) com idade média de $10.04 \pm .06$ anos e estudantes de uma Escola Estadual da cidade de Pelotas/RS. Nenhum participante tinha conhecimento sobre objetivo do experimento e apresentava alteração visual, somatossensorial, auditiva, ou ferimentos que impedissem ou dificultassem a realização dos testes. O presente estudo foi aprovado pelo Comitê de Ética em Pesquisa da Universidade Federal de Pelotas (UFPel) (014/2011) e todos os indivíduos tiveram sua participação concedida após assinatura do Termo de consentimento livre e esclarecido pelos pais ou responsáveis.

\section{Instrumentos}

A tarefa utilizada no estudo foi o teste de agilidade em "L" (Porter et al., 2010), realizado em uma quadra polidesportiva de uma Escola Estadual da cidade de Pelotas/RS. O teste tem se mostrado confiável dado o alto coeficiente de correlação intraclasse $(r=.90)$ encontrado em outros estudos (Gabbett, 2006; Webb \& Lander, 1983). A realização do teste consiste em percorrer cinco metros em linha reta, em seguida cinco metros para a esquerda, contornar um cone e retornar o percurso até a linha de partida, formando um L (Figura 1). A medida utilizada foi o tempo de movimento (TM), mensurado através do cronômetro digital TECHNOS DC453/8P. Apenas um avaliador, previamente treinado, conduziu o teste enquanto outros dois realizaram a tomada dos resultados.

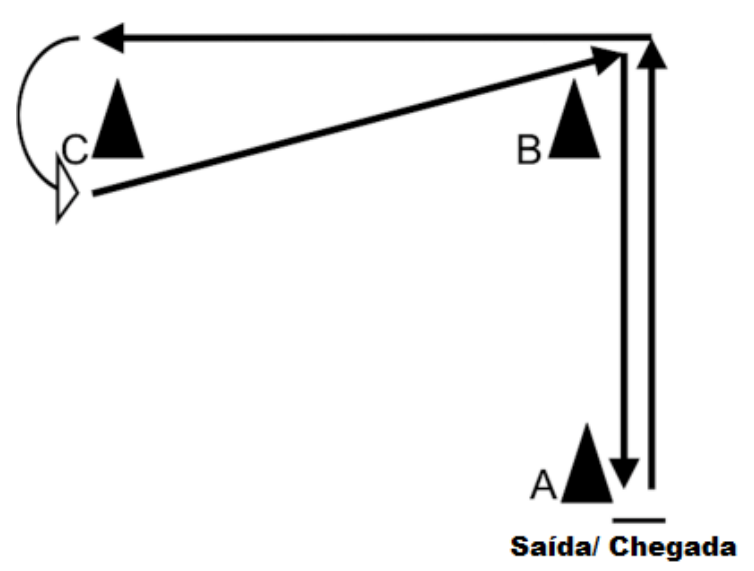

Figura 1. Instrumento de avaliação da agilidade em "L" adaptado de Porter et al. (2010).

\section{Procedimentos}

Cada um dos 23 participantes completou cinco tentativas em cada uma das três condições analisadas: sem foco determinado (condição controle), foco de atenção externo e foco de atenção interno. Como no estudo de Porter et al. (2010) as diferentes condições foram realizadas em dias não consecutivos da semana (segunda, quarta e sexta-feira), sendo ordenados em condição controle no primeiro dia; as condições foco externo e foco interno foram balanceadas no segundo e terceiro dia.

Antes da realização dos testes, os participantes receberam informações, adaptadas de Porter et al. (2010), sobre os procedimentos da tarefa e o tipo de foco de atenção a ser utilizado. $\mathrm{Na}$ condição controle os indivíduos receberam instruções para "correr através do percurso o mais rápido e melhor possível". Na condição de foco de atenção externo foram informados de que deveriam "correr através do percurso o mais rápido e melhor possível, focando nos cones e impulsionando o chão com o pé durante as mudanças de direção do teste". Já na condição de foco interno as crianças receberam instruções de que deveriam "correr através do percurso o mais rápido e melhor possível, focando no movimento das pernas e nos pés durante a execução do teste". 
Os participantes foram conduzidos individualmente até o local de prática e instruídos sobre a realização da tarefa. Para melhor entendimento, o pesquisador demonstrou o percurso a ser realizado de forma lenta e silenciosa afim de não haver influência no foco de atenção instruído ou indiretamente oferecer uma estratégia que poderia ser utilizada para melhorar o desempenho. Em seguida, os participantes foram instruídos a ficar na linha de partida e a começar a tentativa quando estivessem prontos.

O TM começou a ser marcado quando os participantes ultrapassaram a linha de partida e foi finalizado no momento do seu respetivo retorno. Entre as tentativas, cada participante teve um período de descanso de dois minutos, no qual foram convidados a responder à seguinte pergunta: "No que você prestou atenção enquanto corria?”. Em nenhum momento foram autorizados a ver sua resposta anterior. Este procedimento continuou até que todas as cinco tentativas do dia fossem realizadas, não sendo informados o TM ou qualquer outra forma de feedback durante e após as tentativas.

\section{Análise Estatística}

Inicialmente, foram calculadas as médias dos TM apresentados em cada condição para os indivíduos e verificado com o teste $\mathrm{W}$ de ShapiroWilk que os dados diferiam significativamente de uma distribuição normal. Desta forma, foi aplicada a ANOVA de Friedman para verificar diferenças entre as três condições de teste (controle $\mathrm{X}$ foco interno $\mathrm{X}$ foco externo) e testes pareados de Wilcoxon corrigidos pelo procedimento de Bonferroni para identificar as diferenças entre cada condição. Quanto ao nível de significância do teste, foi adotado $5 \%$ para a ANOVA de Friedman e de $1.66 \%$ nas comparações par a par de Wilcoxon. O tamanho de efeito foi calculado a partir da estatística $r$. Para a realização dos procedimentos estatísticos foi utilizado o pacote estatístico SPSS 17.0

As respostas da pergunta em relação ao foco de atenção utilizado foram codificadas e organizadas coletivamente em cada uma das três condições experimentais: foco externo, foco interno e sem foco determinando. A categorização e organização das respostas recebidas, com base no estudo de Porter et al. (2010), foi realizada por dois autores do presente estudo, havendo total concordância na classificação das respostas em cada condição experimental. Por exemplo, quando solicitados para focarem internamente alguns participantes responderam: "prestei atenção no meu pé", "eu corri prestando atenção no movimento dos meus braços". Em contrapartida, respostas como: "prestei atenção nos cones", "corri o mais rápido que pude, olhando para as linhas", foram respostas categorizadas como utilizando o foco de atenção externo.

Além disso, algumas respostas foram classificadas como "outras", no qual os indivíduos misturaram os dois focos de atenção, interno e externo, ou não focaram em nada. Por exemplo, "corri prestando atenção no meu braço, e o mais perto que pude do cone", "não prestei atenção em nada enquanto corria". Estes dados foram analisados a partir da frequência de ocorrência de cada tipo de resposta.

A partir das respostas encontradas, foi verificado se houve associação entre as instruções fornecidas sobre o foco de atenção, o foco adotado e a troca de foco na tentativa seguinte, sendo realizados dois testes de qui-quadrado $\left(\mathrm{X}^{2}\right)$ : condição experimental (foco instruído) $\mathrm{X}$ foco adotado; e condição experimental $\mathrm{X}$ frequência de troca de foco. Para uma análise detalhada, também foram utilizadas a estatística da taxa de razão para verificar em quais condições determinado foco adotado teve probabilidade significativamente superior de ser utilizado em relação outras condições. O mesmo procedimento foi realizado com a análise das trocas de foco.

\section{RESULTADOS}

\section{Tempo de Movimento}

A figura 2 apresenta os desempenhos dos participantes em relação ao TM nos diferentes focos de atenção. A partir da ANOVA de Friedman, foram encontradas diferenças significativas entre as três condições $\left(X^{2}=19.407 ; p=\right.$ 
.001). Nos testes pareados de Wilcoxon identificou-se diferenças significativas entre as condições foco externo e foco interno $(r=.61 ; p=$ .004) e entre as condições foco externo e controle $(\mathrm{r}=.68 ; \mathrm{p}=.001)$ - a condição foco externo resultou em pior desempenho entre as três condições.

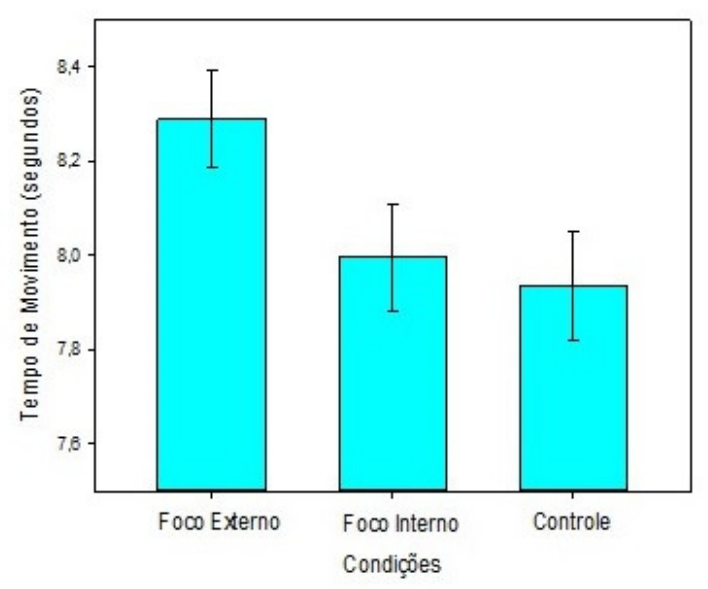

Figura 2. Média e intervalo de confiança (95\%) do tempo de movimento na tarefa de agilidade nas condições de foco externo, interno e controle

\section{Frequência absoluta de respostas relaciona-} das ao foco de atenção utilizado

A tabela 1 apresenta a frequência absoluta de cada resposta sobre o foco de atenção utilizado na execução da tarefa para o questionamento "No que você prestou atenção enquanto corria?”. As condições experimentais apresentaram primazia de foco orientado ao que a condição requeria e distribuição similar entre os outros fo$\cos (63.5 \%$ de foco interno, $19 \%$ foco externo e $17.5 \%$ sem foco determinado na condição foco interno e $61 \%$ de foco externo, $27 \%$ foco interno e $12 \%$ sem foco determinado na condição foco externo). Na condição controle, os participantes apontaram respostas com $65 \%$ de foco externo, $25 \%$ de foco interno e $10 \%$ sem foco determinado.
Sobre a mudança de foco de atenção durante uma mesma condição, os resultados mostram que os participantes alteraram o foco $34 \%$ das vezes na condição de foco interno, $31 \%$ na condição de foco externo, e $17.4 \%$ na condição controle.

\section{Associação entre condição experimental X foco adotado}

A análise de qui - quadrado apontou uma associação significativa entre a condição experimental do indivíduo (foco de atenção instruído) e o foco adotado $\left(x^{2}=61.416 ; p<.001\right)$. A tabela 2 apresenta os resultados relativos a taxa de razão da adoção da instrução do foco em cada condição. Como se pode observar, o foco de atenção foi adotado com maior probabilidade na situação onde este foi instruído (4.92 mais vezes para foco interno e 2.13 mais vezes para foco externo). Adicionalmente, os resultados apontam que houve grande probabilidade de adoção do foco externo na condição controle (2.81 mais vezes) e menor probabilidade do foco interno (.04).

\section{Associação entre condição experimental X troca de foco}

A análise de qui-quadrado apontou uma associação significativa entre a condição experimental do indivíduo (foco de atenção instruído) e o número de trocas de foco $\left(X^{2}=9.093 ; \mathrm{p}=\right.$ .011). A Tabela 3 apresenta os resultados relativos a taxa de razão entre a troca de focos em cada condição. As análises apontam que somente na condição sem foco determinado (controle) observamos resultados significativos. Nesta condição, os participantes apresentaram 2 vezes maior probabilidade de manter o foco escolhido na tentativa seguinte. 
Tabela 1

Frequência absoluta de respostas relacionadas ao foco de atenção utilizado

\begin{tabular}{|c|c|c|c|c|c|c|c|c|c|c|c|}
\hline & \multicolumn{11}{|c|}{ Condição Foco Interno } \\
\hline Categorias & \multicolumn{3}{|c|}{ Externo } & \multicolumn{4}{|c|}{ Interno } & \multicolumn{4}{|c|}{ Outros } \\
\hline Total de & \multicolumn{3}{|c|}{22} & \multicolumn{4}{|c|}{73} & \multicolumn{4}{|c|}{20} \\
\hline $\begin{array}{l}\text { Subcatego- } \\
\text { rias }\end{array}$ & Cones & Chão & $\begin{array}{l}\text { Li- } \\
\text { nhas }\end{array}$ & Pé & $\begin{array}{c}\text { Per } \\
\text { na }\end{array}$ & $\begin{array}{l}\text { Membros } \\
\text { Superiores }\end{array}$ & $\begin{array}{l}\text { Cor- } \\
\text { po }\end{array}$ & $\begin{array}{l}\text { Cur- } \\
\text { vas }\end{array}$ & $\begin{array}{l}\text { Cor- } \\
\text { rer }\end{array}$ & Largada & Nada \\
\hline \multirow[t]{2}{*}{ Respostas } & 16 & 6 & 0 & 48 & 21 & 3 & 1 & 17 & 0 & 0 & 3 \\
\hline & \multicolumn{11}{|c|}{ Condição Foco Interno } \\
\hline Categorias & \multicolumn{3}{|c|}{ Externo } & \multicolumn{4}{|c|}{ Interno } & \multicolumn{4}{|c|}{ Outros } \\
\hline $\begin{array}{l}\text { Total de } \\
\text { respostas }\end{array}$ & \multicolumn{3}{|c|}{70} & \multicolumn{4}{|c|}{31} & \multicolumn{4}{|c|}{14} \\
\hline $\begin{array}{l}\text { Subcatego- } \\
\text { rias }\end{array}$ & Cones & Chão & $\begin{array}{l}\text { Li- } \\
\text { nhas }\end{array}$ & Pé & $\begin{array}{c}\text { Per } \\
\text { na }\end{array}$ & $\begin{array}{l}\text { Membros } \\
\text { Superiores }\end{array}$ & $\begin{array}{l}\text { Cor- } \\
\text { po }\end{array}$ & $\begin{array}{l}\text { Cur- } \\
\text { vas }\end{array}$ & $\begin{array}{l}\text { Cor- } \\
\text { rer }\end{array}$ & $\begin{array}{l}\text { Lar- } \\
\text { gada }\end{array}$ & Nada \\
\hline \multirow[t]{2}{*}{ Respostas } & 20 & 49 & 1 & 24 & 4 & 3 & 0 & 9 & 0 & 2 & 3 \\
\hline & \multicolumn{11}{|c|}{ Condição Controle } \\
\hline Categorias & \multicolumn{3}{|c|}{ Externo } & \multicolumn{4}{|c|}{ Interno } & \multicolumn{4}{|c|}{ Outros } \\
\hline $\begin{array}{l}\text { Total de } \\
\text { respostas }\end{array}$ & \multicolumn{3}{|c|}{75} & \multicolumn{4}{|c|}{29} & \multicolumn{4}{|c|}{11} \\
\hline $\begin{array}{l}\text { Subcatego- } \\
\text { rias }\end{array}$ & Cones & Chão & $\begin{array}{l}\text { Li- } \\
\text { nhas }\end{array}$ & Pé & $\begin{array}{c}\text { Per } \\
\text { na }\end{array}$ & $\begin{array}{l}\text { Membros } \\
\text { Superiores }\end{array}$ & $\begin{array}{l}\text { Cor- } \\
\text { po }\end{array}$ & $\begin{array}{l}\text { Cur- } \\
\text { vas }\end{array}$ & $\begin{array}{l}\text { Cor- } \\
\text { rer }\end{array}$ & $\begin{array}{l}\text { Lar- } \\
\text { gada }\end{array}$ & Nada \\
\hline Respostas & 51 & 20 & 4 & 15 & 8 & 4 & 2 & 5 & 4 & 2 & 0 \\
\hline
\end{tabular}

Tabela 2

Taxa de razão entre Condição experimental x Foco adotado

\begin{tabular}{ccccc}
\hline & \multicolumn{4}{c}{ Foco adotado } \\
\cline { 2 - 4 } & & Externo & Interno & Outros \\
\multirow{4}{*}{ Condições } & Interno & $.13(.08 / .23)^{*}$ & $4.92(3.04 / 7.96)^{*}$ & $1.72(.91 / 3.26)$ \\
& Externo & $2.13(1.35 / 3.36)^{*}$ & $.46(0.28 / .75)^{*}$ & $.88(.45 / 1.74)$ \\
& Controle & $2.81(1.76 / 4.48)^{*}$ & $.40(0.24 / .66)^{*}$ & $.60(1.25 / .29)$ \\
\hline
\end{tabular}

*Valores significativos para $\mathrm{p}<.05$

Tabela 3

Taxa de razão entre Condição experimental $x$ Troca de Focos

\begin{tabular}{cccc}
\hline & \multicolumn{3}{c}{ Troca de Foco } \\
\cline { 2 - 4 } & & Sim & Não \\
Condições & Interno & $1.60(.98 / 2.62)$ & $.62(.38 / 1.01)$ \\
& Externo & $1.32(.81 / 2.17)$ & $.75(0.45 / 1.23)$ \\
& Controle & $.42(.24 / .74)^{*}$ & $2.32(1.33 / 4.05)^{*}$ \\
\hline
\end{tabular}

* Valores significativos para $\mathrm{p}<.05$

\section{DISCUSSÃO}

No presente estudo foi investigado se instruções de foco de atenção externo comparado ao foco interno e sem foco determinando afetariam de forma diferente a performance motora de crianças. Ainda, o estudo analisou a adoção da instrução verificando qual foco de atenção foi utilizado pelos participantes.
Observando os desempenhos das condições experimentais, as análises apontaram diferenças no tempo de movimento entre os diferentes focos de atenção induzidos: as condições controle e com instrução de foco interno apresentaram desempenhos melhores que a condição de foco externo. Tais resultados assemelham-se aos achados verificados na fase de desempenho dos 
estudos de foco de atenção e aprendizagem motora em crianças (Abdollahipour et al., 2011; Chiviacowsky et al., 2013; Wulf, Chiviacowsky, et al., 2010), demonstrando que instruções dirigindo a atenção de crianças para foco externo, ao invés de nenhum foco determinado ou foco interno, não resultaram em uma performance mais eficaz.

Esses resultados podem estar relacionados aos efeitos do foco de atenção em distintos níveis de aprendizagem. Alguns estudos demonstraram diferenças em mecanismos de atenção de participantes iniciantes e habilidosos, demonstrando que adultos em estágios iniciais de aquisição de habilidades têm benefícios na performance motora com a utilização do foco interno de atenção (Beilock et al., 2004, 2002; Ford et al., 2005).

Os resultados do presente estudo corroboram com a ideia de que no desempenho de iniciantes, não automático, a execução de habilidades é suportada por um conjunto de estruturas de controlo que são mantidos na memória de trabalho, não podendo ser rompida através do controle consciente e, dessa forma, facilitando os processos iniciais de habilidade (Beilock et al., 2002; Ford et al., 2005). Logo, durante os primeiros estágios de aprendizagem, existe influência positiva da direção da atenção nos padrões de movimento, não sendo estas tão relevantes para indivíduos habilidosos (Schmidt \& Lee, 2011).

Aliado a isto, alguns estudos analisando os efeitos do foco de atenção na aprendizagem motora de adultos iniciantes demonstraram que, ao receber informações de foco interno na instrução inicial e/ou nas primeiras tentativas de prática e somente após um período de prática receber instrução de foco externo, obtiveram melhor aprendizagem (Oliveira, Denardi, Tani, \& Corrêa, 2013; Silva et al., 2013). Assumindo que a performance motora de crianças seria semelhante, ou até inferior, a de adultos iniciantes, estes resultados destacam diferenças no desempenho das estruturas de controlo de atenção em distintos níveis de aprendizagem e complementam um crescente corpo de estudos que sugere que a performance de indivíduos iniciantes não é beneficiada pelo foco externo de atenção.

Em relação à adoção do foco de atenção, os resultados apontaram que as crianças se utilizaram prioritariamente dos focos de atenção instruídos pelo experimentador. As condições com instrução direta tiveram $61 \%$ e $63.5 \%$ das vezes com as crianças utilizando o foco instruído, sendo verificada associação significativa entre a condição experimental do indivíduo (foco de atenção instruído) e o foco adotado. Este nível de adoção da instrução foi encontrada também em adultos na mesma tarefa (Porter et al., 2010). A quantidade de trocas de foco nestas condições também foram similares ao estudo de Porter et al. (2010). Entretanto, os participantes na condição sem foco determinado focaram atenção externamente em $65 \%$ das tentativas e escolheram mudar seu foco de atenção em uma frequência de $17.4 \%$, demonstrando, em análises adicionais, que as crianças significativamente apresentaram 2 vezes maior probabilidade de manter o foco escolhido na tentativa seguinte. Estes resultados diferem dos resultados de Porter et al. (2010) - neste estudo o foco para a condição controle é disperso e as trocas de foco são mais frequentes - e se apresentam contraditórios aos resultados da condição de foco externo.

Uma explicação para os resultados apresentados em cada condição pode estar relacionada ao conteúdo da instrução do foco induzido. Ao visualizarmos a frequência absoluta de cada resposta para a questão do foco adotado, observam-se diferenças nas subcategorias do foco de atenção externo utilizado: a condição de foco externo teve maioria de tentativas com foco no chão enquanto a condição controle teve maioria nos cones (49 e 51 vezes respetivamente). Esses resultados apontam que o conteúdo do foco de atenção prescrito para a condição de foco externo não foi eficiente para realização da tarefa; as informações relacionadas ao foco de atenção fornecidas seriam inespecíficas.

Alguns autores (por exemplo, Ried, Fugita, Freudenheim, Basso, \& Corrêa, 2012; Schmidt 
\& Lee, 2011; Wulf, 2013) têm destacado a influência e implicações da formulação e adoção da instrução do foco de atenção. Por exemplo, Ried, Fugita, Freudenheim, Basso, e Corrêa (2012) argumentam que é possível que fatores como a linguagem utilizada, definida pelo contexto, pelo tema e pelos falantes, dificultem a compreensão da instrução na realização das tarefas por parte de alguns dos executantes. Schimdt \& Lee (2011) complementam que é muito importante que a instrução seja clara, concisa e repetida várias vezes para encorajar os indivíduos a centrar sua atenção no ponto desejado. Se faz necessário, portanto, compreender que instruções sobre foco devem considerar pontos relevantes da tarefa - que possam influenciar de forma positiva o desempenho dos executantes.

Desta forma, se considerarmos que os focos induzidos no presente estudo implicaram em dois aspetos a serem focalizados ao mesmo tempo, podemos supor que essa seja uma limitação do presente estudo. A consideração deste aspeto pode estar intimamente ligada à quantidade de informações presentes na instrução e a relação com a capacidade de processamento de informações (Badan, Hauert, \& Mounoud, 2000; M. T. H. Chi, 1977; Connolly, 1970; Lambert \& Bard, 2005). Especificamente, crianças se encontram em um nível maturacional anterior em relação aos adultos, o que compromete o processo de avaliação do erro afetando a performance motora (Chi, 1976; Thomas, 1980). Thomas (1980) ressalta ainda diferenças na utilização de processos de controlo (ensaio ou prática, rotulação, procura e resgate, agrupamento e codificação) para transferir a informação de memória de curta duração para memória de longa duração, sendo as crianças mais lentas no processamento.

Observa-se assim, que o foco de atenção bem-sucedido em adultos (Porter et al., 2010) não foi superior para as crianças do presente estudo. Ao levar em conta a colocação de Thomas, Lee, \& Thomas (1988), que classificam as crianças como "novatos universais" devido a quantidade limitada de experiências que elas possuem, percebe-se que a falta de experiências podem refletir diretamente na performance e na influência do foco utilizado.

Deve ser enfatizado que este estudo reforça a necessidade de incluir instrumentos de controlo da adoção do foco em pesquisas relativas à eficiência do foco de atenção e a importância do conteúdo fornecido para instrução do foco a ser utilizado. Assim, é esperado que outros estudos sejam realizados para a compreensão dos possíveis efeitos do foco de atenção em crianças, utilizando testes de retenção e transferência em diferentes tarefas e condições metodológicas a fim de verificar efeitos permanentes na performance e aprendizagem motora.

\section{CONCLUSÕES}

Conclui-se que a utilização de instrução de foco externo de atenção não beneficiou a performance motora de crianças. Pode-se argumentar que as diferenças decorrentes dos níveis de aprendizagem não possibilitam observar o mesmo efeito do foco de atenção observado em adultos. Verifica-se ainda, que as crianças se utilizaram prioritariamente dos focos de atenção instruídos pelo experimentador. Entretanto, a utilização de foco de atenção quando não há indução por parte do experimentador é prioritariamente de foco externo.

\section{Agradecimentos: \\ Nada a declarar.}

\section{Conflito de Interesses:}

Nada a declarar.

\section{Financiamento:}

Cnpq/Brasil- Protocolo 211487/2013-9.

\section{REFERÊNCIAS}

Abdollahipour, R., Bahram, A., Shafizadeh, M., \& Khalaji, H. (2011). The effects of attentional focus strategies on the performance and learning 
of soccer-dribbling task in children and adolescences. Journal of Movement Sciences \& Sports, (Special Issue 1), 83-92.

Abdollahipour, R., Bahram, A., Shafizadeh, M., \& Khalaji, H. (2011). The effects of attentional focus strategies on the performance and learning of soccer-dribbling task in children and adolescences. Journal of Movement Sciences \& Sports, (Special Issue 1), 83-92.

Badan, M., Hauert, C. A., \& Mounoud, P. (2000). Sequential pointing in children and adults. Journal of Experimental Child Psychology, 75(1), 4369. http://doi.org/10.1006/jecp.1999.2522

Beilock, S. L., Bertenthal, B. I., Mccoy, A. M., \& Carr, T. H. (2004). Haste does not always make waste: Expertise, direction of attention, and speed versus accuracy in performing sensorimotor skills. Psychonomic Bulletin \& Review, 11(2) 373-379. http://doi.org/10.3758/BF03196585

Beilock, S. L., Carr, T. H., MacMahon, C., \& Starkes, J. L. (2002). When paying attention becomes counterproductive: Impact of divided versus skill-focused attention on novice and experienced performance of sensorimotor skills. Journal of Experimental Psychology: Applied, 8(1), 6-16. http://doi.org/10.1037/1076-898X.8.1.6

Bell, J. J., \& Hardy, J. (2009). Effects of Attentional Focus on Skilled Performance in Golf. Journal of Applied Sport Psychology, 21(2), 163-177. http://doi.org/10.1080/10413200902795323

Chi, M. T. (1976). Short-term memory limitations in children: Capacity or processing deficits? Memory \& Cognition, 4(5), 559-572. http://doi.org/10.3758/BF03213219

Chi, M. T. H. (1977). Age differences in memory span. Journal of Experimental Child Psychology, 23(2), 266-281. http://doi.org/10.1016/00220965(77)90104-7

Chiviacowsky, S., Wulf, G., \& Avila, L. T. G. (2013). An external focus of attention enhances motor learning in children with intellectual disabilities. Journal of Intellectual Disability Research: JIDR, 577(7), 627-634. http://doi.org/10.1111/j.13652788.2012.01569.x

Connolly, K. J. (Ed.). (1970). Mechanisms of Motor Skill Development (Study Group on Mechanisms of Motor Skill Development, Centre for Advanced Study in the Developmental Sciences, Ciba Foundation). London: Academic Press.

Emanuel, M., Jarus, T., \& Bart, O. (2008). Effect of focus of attention and age on motor acquisition, retention, and transfer: a randomized trial. Physical Therapy, 88(2), 251-260. http://doi.org/10.2522/ptj.20060174

Fitts, P. M., \& Posner, M. I. (1967). Human performance. Belmont: Brooks.
Ford, P., Hodges, N. J., \& Williams, A. M. (2005). Online attentional-focus manipulations in a soccer-dribbling task: implications for the proceduralization of motor skills. Journal of Motor Behavior, 37(5), 386-394. http://doi.org/10.3200/JMBR.37.5.386-394

Ford, P., Hodges, N. J., \& Williams, A. M. (2009). An evaluation of end-point trajectory planning during skilled kicking. Motor Control, 13(1), 1-24.

Freudenheim, A. M., Wulf, G., Madureira, F., Pasetto, S. C., \& Corrêa, U. C. (2010). An external focus of attention results in greater swimming speed. International Journal of Sports Science \& Coaching, 5(4), 533-542. http://doi.org/10.1260/1747-9541.5.4.533

Gabbett, T. J. (2006). Skill-based conditioning games as an alternative to traditional conditioning for rugby league players. Journal of Strength and Conditioning Research, 20(2), 309-315. http://doi.org/10.1519/R-17655.1

Gentile, A. M. (1972). A Working Model of Skill Acquisition with Application to Teaching. Quest, $17(1)$,

3-23. http://doi.org/10.1080/00336297.1972.10519 717

Ivry, R. (1996). Chapter 5 Representational issues in motor learning: Phenomena and theory. Em H. Heuer \& S. W. Keele (Eds.), Handbook of Perception and Action ( $2^{\mathrm{a}}$ ed., Vol. 2, pp. 263-330). London: Academic Press.

Kal, E. C., van der Kamp, J., \& Houdijk, H. (2013). External attentional focus enhances movement automatization: a comprehensive test of the constrained action hypothesis. Human Movement Science, 32(4), 527-539. http://doi.org/10.1016/j.humov.2013.04.001

Lambert, J., \& Bard, C. (2005). Acquisition of visuomanual skills and improvement of information processing capacities in 6- to 10-year-old children performing a $2 \mathrm{D}$ pointing task. Neuroscience Letters, 377(1), 1-6. http://doi.org/10.1016/j.neulet.2004.11.058

Lohse, K. R., Sherwood, D. E., \& Healy, A. F. (2010). How changing the focus of attention affects performance, kinematics, and electromyography in dart throwing. Human Movement Science, 29(4), 542-555. http://doi.org/10.1016/j.humov.2010.05.001

Makaruk, H., Porter, J. M., Czaplicki, A., Sadowski, J., \& Sacewicz, T. (2012). The role of attentional focus in plyometric training. The Journal of Sports Medicine and Physical Fitness, 52(3), 319-327.

Marchant, D. C., Greig, M., Bullough, J., \& Hitchen, D. (2011). Instructions to adopt an external focus enhance muscular endurance. Research Quarterly for Exercise and Sport, 82(3), 466473. http://doi.org/10.1080/02701367.2011.10599 779 
Oliveira, T. A. C., Denardi, R. A., Tani, G., \& Corrêa, U. C. (2013). Effects of Internal and External Attentional Foci on Motor Skill Learning: Testing the Automation Hypothesis. Human Movement, 14(3), 194-199. http://doi.org/10.2478/humo-2013-0022

Olivier, I., Palluel, E., \& Nougier, V. (2008). Effects of attentional focus on postural sway in children and adults. Experimental Brain Research, 185(2), 341-345. http://doi.org/10.1007/s00221-008-1271-6

Porter, J. M., Nolan, R. P., Ostrowski, E. J., \& Wulf, G. (2010). Directing attention externally enhances agility performance: a qualitative and quantitative analysis of the efficacy of using verbal instructions to focus attention. Frontiers in Psychology, $1, \quad 216$. http://doi.org/10.3389/fpsyg.2010.00216

Ried, B., Fugita, M., Freudenheim, A. M., Basso, L., \& Corrêa, U. C. (2012). Instrução verbal: solicitar foco de atenção não garante sua adoção e melhor desempenho. Motriz, 18(3), 449-455. http://doi.org/10.1590/S198065742012000300005

Schlesinger, M., Porter, J., \& Russell, R. (2012). An external focus of attention enhances manual tracking of occluded and visible targets. Frontiers in Psychology, 3, 591. http://doi.org/10.3389/fpsyg.2012.00591

Schmidt, R. A., \& Lee, T. (2011). Motor Control and Learning: A Behavioral Emphasis (5 ${ }^{\text {a }}$ ed.). Champagn, IL: Human Kinetics.

Schücker, L., Hagemann, N., Strauss, B., \& Völker, K. (2009). The effect of attentional focus on running economy. Journal of Sports Sciences, 27(12),

1241-1248. http://doi.org/10.1080/02640410903150467

Shiffrin, R. M. (1988). Attention. Em R. Atkinson, R. Herrnstein, G. Lindzey, \& R. Luce (Eds.), Stevens' Handbook of Experimental Psychology (pp. 739-811). New York: Wiley.
Silva, C. B. M., Benda, R. N., Fonseca, F. de S., Fialho, J. V. A. P., Menzel, H.-J. K., \& Ugrinowitsch, H. (2013). Mudança no foco de atenção ao longo da prática de uma habilidade motora. Motriz, 19(2), 391-398. http://doi.org/10.1590/S198065742013000200016

Stoate, I., \& Wulf, G. (2011). Does the Attentional Focus Adopted by Swimmers Affect Their Performance? International Journal of Sports Science and Coaching, 6(1), 99-108. http://doi.org/10.1260/1747-9541.6.1.99

Thomas, J. R. (1980). Acquisition of motor skills: information processing differences between children and adults. Research Quarterly for Exercise and Sport, 51(1), 158-173. http://doi.org/10.1080/02701367.1980.10609 281

Thomas, J. R., Lee, A. M., \& Thomas, K. T. (1988). Physical Education for Children: Concepts into Practice. Champaign, IL: Human Kinetics Pub.

Webb, P., \& Lander, J. (1983). An economical fitness testing battery for high school and college rugby teams. Sports Coach, 7, 44-46.

Wulf, G. (2007). Attention and Motor Skill Learning. Champagn, IL: Human Kinetics.

Wulf, G. (2013). Attentional focus and motor learning: a review of 15 years. International Review of Sport and Exercise Psychology, 6(1), 77-104. http://doi.org/10.1080/1750984X.2012.72372 8

Wulf, G., Chiviacowsky, S., Schiller, E., \& Avila, L. T. G. (2010). Frequent external-focus feedback enhances motor learning. Frontiers in Psychology, 1, http://doi.org/10.3389/fpsyg.2010.00190

190.

Wulf, G., Dufek, J. S., Lozano, L., \& Pettigrew, C. (2010). Increased jump height and reduced EMG activity with an external focus. Human Movement Science, 29(3), 440-448. http://doi.org/10.1016/j.humov.2009.11.008 